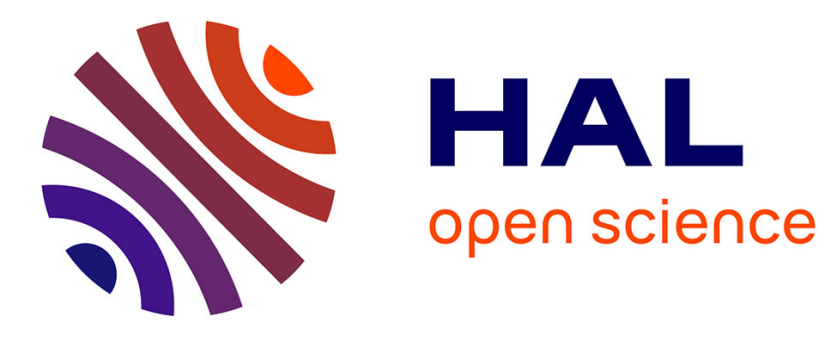

\title{
LiHe spectra from brown dwarfs to helium clusters
}

Nicole F. Allard, Akira Nakayama, Frank Stienkemeier, John F. Kielkopf, Grégoire Guillon, Alexandra Viel

\section{To cite this version:}

Nicole F. Allard, Akira Nakayama, Frank Stienkemeier, John F. Kielkopf, Grégoire Guillon, et al.. LiHe spectra from brown dwarfs to helium clusters. Advances in Space Research, 2014, 54, pp.12901296. 10.1016/j.asr.2013.08.032 . hal-00876511

\section{HAL Id: hal-00876511 https://hal.science/hal-00876511}

Submitted on 25 Oct 2013

HAL is a multi-disciplinary open access archive for the deposit and dissemination of scientific research documents, whether they are published or not. The documents may come from teaching and research institutions in France or abroad, or from public or private research centers.
L'archive ouverte pluridisciplinaire HAL, est destinée au dépôt et à la diffusion de documents scientifiques de niveau recherche, publiés ou non, émanant des établissements d'enseignement et de recherche français ou étrangers, des laboratoires publics ou privés. 


\title{
LiHe Spectra From Brown Dwarfs to Helium Clusters
}

\author{
N.F. Allard ${ }^{1, *}$ \\ Observatoire de Paris, GEPI, UMR 8111, CNRS, Université Denis Diderot, 61, Avenue de l'Observatoire, F-75014, Paris, France
}

A. Nakayama*

Division of Chemistry, Graduate School of Science, Hokkaido University, Sapporo 060-0810, Japan

F. Stienkemeier*

Physikalisches Institut, Universität Freiburg, Hermann-Herder-Str. 3, D-76104 Freiburg, Germany

J.F. Kielkopf*

Department of Physics and Astronomy, University of Louisville, Louisville, Kentucky 40292 USA

G. Guillon and A. Viel*

Institut de Physique de Rennes, UMR 6251, CNRS et Université de Rennes 1, F-35042 Rennes, France

\begin{abstract}
The detection of Li I lines is the most decisive spectral indicator of substellarity for young brown dwarfs with masses below about 0.06 solar mass. Due to the weakness of the Li resonance lines, it is important to be able to model precisely both their core widths and their wing profiles. This allows an adequate prediction of the mass at which Li lines reappear in the spectra of brown dwarfs for a given age, or reversely an accurate determination of the age of a cluster. We report improved line profiles and the dependence of line width on temperature suitable for modeling substellar atmospheres that were determined from new LiHe molecular potential energies. Over a limited range of density and temperature, comparison with laboratory measurements was used to validate the potential energies which support the spectral line profile theory.
\end{abstract}

Keywords: Line: profiles; Stars: atmospheres-Brown dwarfs; Atomic and molecular clusters

\section{Introduction}

Alkali metals are the last optical opacity source to condense out to dust grains in cool substellar atmospheres. Their resonance lines provide a pseudo-continuum that shapes the emitted spectrum from the UV to the nearinfrared spectral range. Model atmosphere, synthetic spectra, and color predictions rely therefore on an adequate treatment of the far wings of alkali resonance lines in the presence of high densities of $\mathrm{H}_{2}$ and He perturbers. Detailed knowledge of the line profiles as a function of temperature and pressure can be obtained from semi-classical calculations using accurate molecular potential energy curves

\footnotetext{
*Corresponding author N.F. Allard

Email addresses: nicole.allard@obspm.fr (N.F. Allard), akira-n@sci.hokudai.ac.jp (A. Nakayama),

stienkemeier@uni-freiburg.de (F. Stienkemeier),

kielkopf@louisville.edu (J.F. Kielkopf), elgringgo@gmail.com

and Alexandra.Viel@univ-rennes1.fr (G. Guillon and A. Viel)

${ }^{1}$ Institut d'Astrophysique de Paris, UMR7095, CNRS, Université Pierre et Marie Curie, 98bis Boulevard Arago, F-75014, Paris, France
}

and dipole transition moments for the alkali-perturber system. The line profiles can then be used as valuable diagnostics of the atmospheres of brown dwarfs.

In Allard et al. (2005) we presented the absorption profiles of lithium perturbed by helium using molecular potential energies of Pascale (1983). Similar profiles in the Li-He line wings were obtained by Zhu et al. (2005) and Alioua and Bouledroua (2006) in full quantum mechanical calculations in the binary approximation. Line parameters of the light alkalis perturbed by helium have been obtained by Peach et al. (2006) and Mullamphy et al. (2007) from a close-coupling description of the colliding atoms, the Baranger theory for collision broadening, and ab initio potential energies described by Mullamphy et al. (2007). Impact broadening of light alkali lines computed in a semiclassical approach was studied in Allard et al. (2007). 


\section{New molecular potential energies and resulting profiles}

We consider the absorption spectrum of the $\mathrm{Li}(2 s-2 p)$ resonance transition in the presence of helium for the physical conditions of the atmosphere of brown dwarf stars. A unified theory of spectral line broadening has been developed to calculate neutral atom spectra given the interaction and radiative transition moments for relevant states of the radiating atom with other atoms in its environment. Our approach is based on the quantum theory of line shapes of Baranger (1958b,a) developed in an adiabatic representation to include the degeneracy of atomic levels (Royer, 1974, 1980; Allard et al., 1994). Complete details and the derivation of the theory are given by Allard et al. (1999).

\subsection{Li-He diatomic potential energies without spin-orbit coupling}

Because line wing intensities are functions of the excited and ground state interactions, knowledge of the electronic energies is of great importance to achieve accurate evaluations of the line profiles. Molecular-structure calculations which neglect the spin-orbit interaction have been performed by Pascale (1983) to obtain the adiabatic potential energies of alkali-He systems, and Behmenburg et al. (1996) obtained ab initio adiabatic potential energies for excited states. Very recently, the ground state and the first two excited states of $\mathrm{Li}-\mathrm{He}$ and $\mathrm{Na}-\mathrm{He}$ have been determined using high level $a b$ initio methods, namely, state-averaged complete active space self-consistent fieldmultireference configuration interaction (SA-CASSCF-MRCI) All $a b$ initio calculations were performed using the MOLPRO 2006.1 package $^{2}$. They were obtained over a large range of intermolecular distances allowing a good description of the spectral line core. This is crucial to interpret absorption spectra of Li obtained at low temperature (Section 5). Complete details are given by Dell'Angelo et al. (2012).

However, an important question to address is whether the small value of the Russell-Saunders-like spin-orbit interaction for the $\mathrm{Li}$ atom has to be taken into account to obtain accurate spectral line profiles. This value $\left(\Delta_{S O}=\right.$ $0.34 \mathrm{~cm}^{-1}$ in ${ }^{2} \mathrm{P}$ state) is only of the order of magnitude of the accuracy of the energies obtained within the framework of methods and extended basis sets described in Dell'Angelo et al. (2012). As a result, we believe this effect may be best accounted for if the value of the atomic Li spin-orbit coupling parameter $\Delta_{S O}$ varies slowly when the $\mathrm{Li}$ and He atoms approach one another. This assumption seems entirely reasonable, given the generally weak interaction between Li and He. No spin-orbit matrix elements, on the basis of the Breit-Pauli operator, were computed $a b$ initio as a function of the internuclear separation, but

\footnotetext{
${ }^{2}$ http://www.molpro.net
}

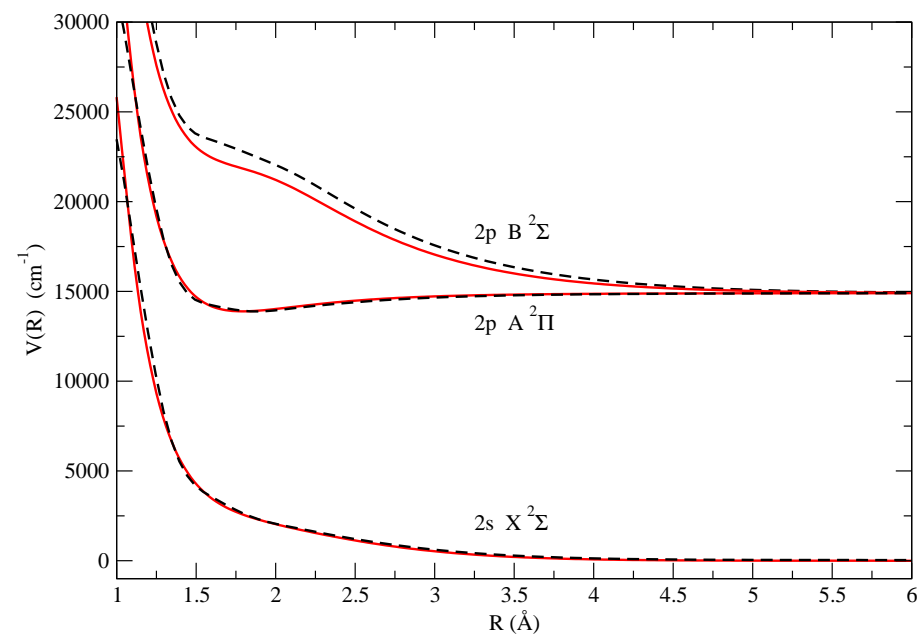

Figure 1: Potential energy curves for the low-lying $X, A$ and $B$ states of Li-He (Dell'Angelo et al., 2012) without spin-orbit coupling (all red lines). The Li-He pseudo-potentials (all black lines) of (Pascale, 1983) are superimposed.

they were supposed to be reasonably constant. In this way, changes in $\Delta_{S O}$ are almost entirely due to the recoupling of angular momenta between the situation of a molecule with axial symmetry when the atoms are close, compared to two separated spherical atoms (Cooper, 1982). Furthermore, the procedure of using asymptotic atomic spin-orbit parameters to obtain more accurate potential energy curves has been shown to be essential for a proper understanding of spectroscopic observations of weakly interacting sys). tems involving rare gas atoms (Cohen and Schneider, 1974; Schneider and Cohen, 1974).

In Fig. 1 we show the energies of the $2 s-2 p$ states of the $\mathrm{Li}-\mathrm{He}$ system neglecting spin-orbit coupling. Li-He potentials computed taking into account spin-orbit coupling are presented in Section 5.1.

\subsection{Absorption spectra for brown dwarfs}

Blue satellite bands in alkali-He $/ \mathrm{H}_{2}$ profiles can be predicted from the extrema in the difference potential energies $\Delta V(R)$, given by

$$
\Delta V(R) \equiv V_{e^{\prime} e}[R(t)]=V_{e^{\prime}}[R(t)]-V_{e}[R(t)],
$$

which represents the difference between the electronic energies of the quasi-molecular $e^{\prime}-e$ transition. The potential energy for a state $e$ is

$$
V_{e}[R(t)]=E_{e}[R(t)]-E_{e}^{\infty} .
$$

The difference in the $B$ state between the new ab initio potential energies and the calculated pseudopotential energies shown in Fig. 1 affects the blue $B-X$ satellite position. Figure 2 shows that the $B-X$ difference potential energies for Li-He go through a maximum, $\Delta V_{m} \sim 5300 \mathrm{~cm}^{-1}$ for Pascale (1983) and $\sim 4400 \mathrm{~cm}^{-1}$ for Dell'Angelo et al. $(2012)$ at a similar small internuclear separation $\left(R_{m} \sim\right.$ 


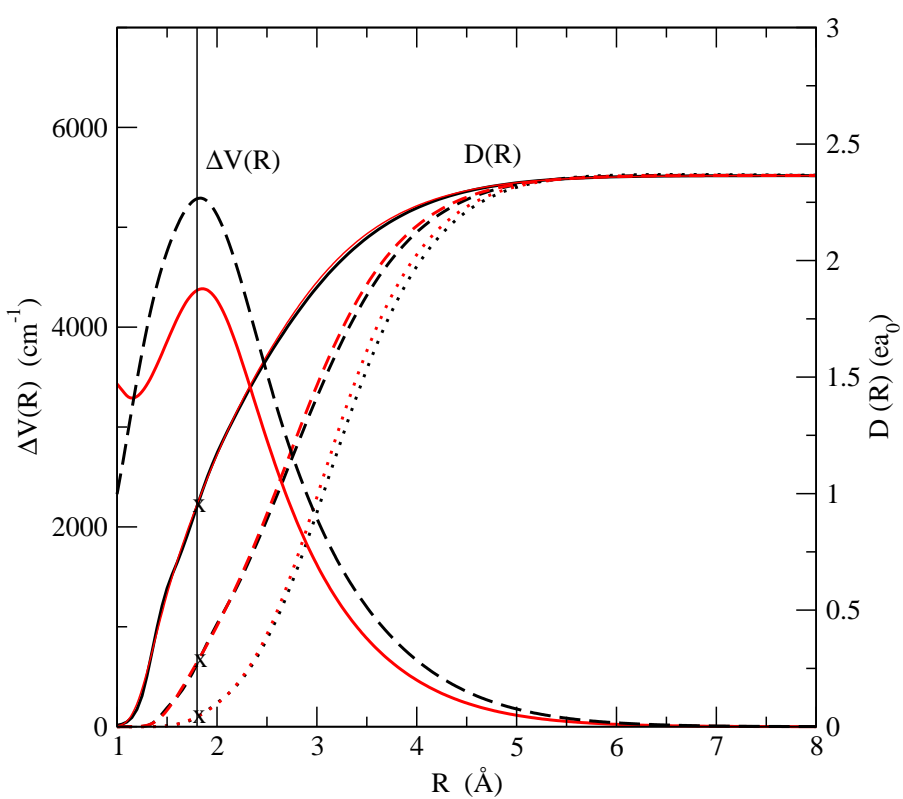

Figure 2: Comparison of $B^{2} \Sigma-X^{2} \Sigma$ difference potential energies and modulated dipole moments at $3000 \mathrm{~K}$ (solid line), $1000 \mathrm{~K}$ (dashed lines) and $500 \mathrm{~K}$ (dotted lines). Dell'Angelo et al. (2012)(all red lines), Pascale (1983)(all black lines). The vertical line at $R=R_{m}$ indicates the position of the maximum of $\Delta V(\mathrm{R})$.

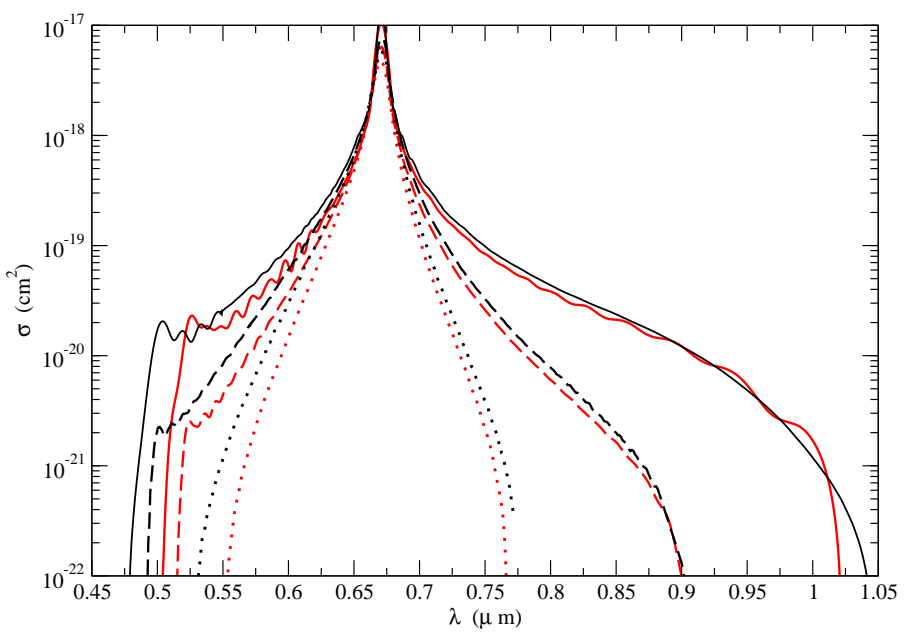

Figure 3: Variation of the line profiles of $\mathrm{Li}$ perturbed by He with temperature. The density of perturbers is $n_{\mathrm{He}}=10^{19} \mathrm{~cm}^{-3}$. The temperatures are $3000 \mathrm{~K}$ (solid line), $1000 \mathrm{~K}$ (dashed lines) and $500 \mathrm{~K}$ (dotted lines). Absorption spectra (all black lines Pascale (1983), all red lines Dell'Angelo et al. (2012)).
$1.8 \AA$ ), the line satellite being predominantly due to perturbers at this distance. The corresponding line satellite shown in Fig. 3 is at $0.526 \mu \mathrm{m}$ for Dell'Angelo et al. (2012) and at $0.5 \mu \mathrm{m}$ for Pascale (1983).

The average number of perturbers in the interaction volume at $R_{m}$ is the determining parameter for the amplitude of the satellites on the spectral line (Allard, 1978; Royer, 1978). Consequently, the satellite amplitude will increase as $R_{m}^{2}$. It also depends on the value of the electric dipole transition moment in the internuclear region where the line satellite is formed (Allard et al., 1998). For a transition $\alpha=(i, f)$ from initial state $i$ to final state $f$, we defined $\tilde{d}_{e e^{\prime}}(R(t))$ as a modulated dipole (Eq. 117 of Allard et al. (1999))

$$
D(R) \equiv \tilde{d}_{e e^{\prime}}[R(t)]=d_{e e^{\prime}}[R(t)] e^{-\frac{\beta}{2} V_{e}[R(t)]},
$$

where $\beta$ is the inverse temperature $(1 / k T)$, and $e$ and $e^{\prime}$ label the electronic energy on which the interacting atoms approach the initial and final atomic states of the transition as $R \rightarrow \infty$. Here $V_{e}$ is the ground state potential energy because we consider the absorption spectrum of the resonance transition. Over regions where $V_{e}(r)<0$, the factor $e^{-\beta V_{e}(r)}$ accounts for bound states of the radiatorperturber pair, but in a classical approximation wherein the discrete bound states are replaced by a continuum. Thus any band structure is smeared out.

Fig. 2 shows the modulated dipole moments $D(R)$ for different temperatures, together with the corresponding $\Delta V(R)$ for potential energies calculated by Pascale (1983) and by Dell'Angelo et al. (2012). In both cases we use for the transition moments the $d_{e e^{\prime}}$ of Pascale (1983), and the difference between the corresponding $D(R)$ (black and red curves) is due only to $V_{e}$. In this range of temperature and distance, the new potentials do not produce a very significant change in $D(R)$. However, Fig. 3 shows clearly the presence of a far blue satellite that has a wavelength of about $0.5 \mu \mathrm{m}$ using the pseudopotential energies of Pascale (1983), and about $0.48 \mu \mathrm{m}$ for ab initio potential energies of Dell'Angelo et al. (2012). This satellite has about the same amplitude in both cases. The red wing is unchanged becaused the potential energies for the $\mathrm{X}^{2} \Sigma^{+}$ and $\mathrm{A}^{2} \Pi$ states shown in Fig. 1 are similar in both theoretical models.

The appearance of the blue satellite is also very sensitive to temperature due to the fast variation of the modulated dipole moment $D$ with temperature in the region of the maximum of $\Delta V$. As shown in Fig. 2, $D\left(R_{m}\right)$ is almost zero for $\mathrm{T}=500 \mathrm{~K}$ and the $B-X$ satellite has totally disappeared at the lowest temperatures considered here (Fig. 3).

\section{Impact line parameters}

The $\lambda 0.671 \mu \mathrm{m}$ doublet is located in a part of an otherwise busy optical spectrum where it can emerge from the molecular pseudo-continuum despite its shallow strength 
(compared to potassium or sodium doublets). To predict this growth, it is important to be able to model precisely both the core width and the wing profiles of the lines. This permits a determination of the brown dwarf mass at which Li lines reappear in their spectra for a given age, or conversely an accurate determination of the age of a cluster from $\mathrm{Li}$ absorption line strengths.

The impact theories of pressure broadening (Baranger, $1958 \mathrm{~b}, \mathrm{a})$ are based on the assumption of sudden collisions (impacts) between the radiator and perturbing atoms, and are valid when frequency displacements $\Delta \omega=\omega-\omega_{0}$ and gas densities are sufficiently small. The usefulness of the impact approximation lies in its simplicity and especially in the fact that its characteristic shift and width have a dependence on density and temperature that may be calculated easily for sufficiently low density when the atomic interaction potential energies are known. Since we are considering the low pressure involved in the upper atmosphere of brown dwarfs, we might expect that the impact approximation will be a good starting point for synthetic spectra. It produces a Lorentzian line with a width and shift linearly dependent on density, and usually a simple power low dependence on temperature. We distinguish a line core as the region of a few halfwidths of the line center over which the impact theory is adequate. Because the core can be opaque, it is important to realize that the line wings, far from the core, are often not well described by the impact approximation.

Calculations have been done for the $D 1$ and $D 2$ lines of $\mathrm{Li}$ from $400 \mathrm{~K}$ to $4000 \mathrm{~K}$, using the $a b$ initio potential energies of Dell'Angelo et al. (2012) shown in detail in Figs. 6 and 7. In Table 1 we report our computed halfwidths at half maximum (HWHM) obtained in the semiclassical $(s c)$ theory. We also report for comparison those derived from the power law form of the close coupled $(c c)$ calculations of Peach et al. (2006). In their work, line parameters of the light alkalis perturbed by helium have been obtained from a close-coupling description of the colliding atoms, the Baranger theory for collision broadening, and previous ab initio potential energies of Mullamphy et al. (2007). The line widths $w$ (HWHM) are linearly dependent on He density, and a power law in temperature given for the ${ }^{2} P_{1 / 2}$ transition by

$$
w=0.067307 \times 10^{-20} n T^{0.40453}
$$

and for the ${ }^{2} P_{3 / 2}$ transition by

$$
w=0.094943 \times 10^{-20} n T^{0.39382}
$$

The non-linear dependence on temperature is illustrated in Fig.4. These expressions may be used to compute the widths for temperatures of stellar or planetary atmospheres from 500 to at least $4000 \mathrm{~K}$.

There are several independent measurements of the broadening of the $\mathrm{Li}$ resonance lines by $\mathrm{He}$ reported in the literature, and we have summarized them in Table 2. We note that the shifts $(d)$ are a small fraction of the

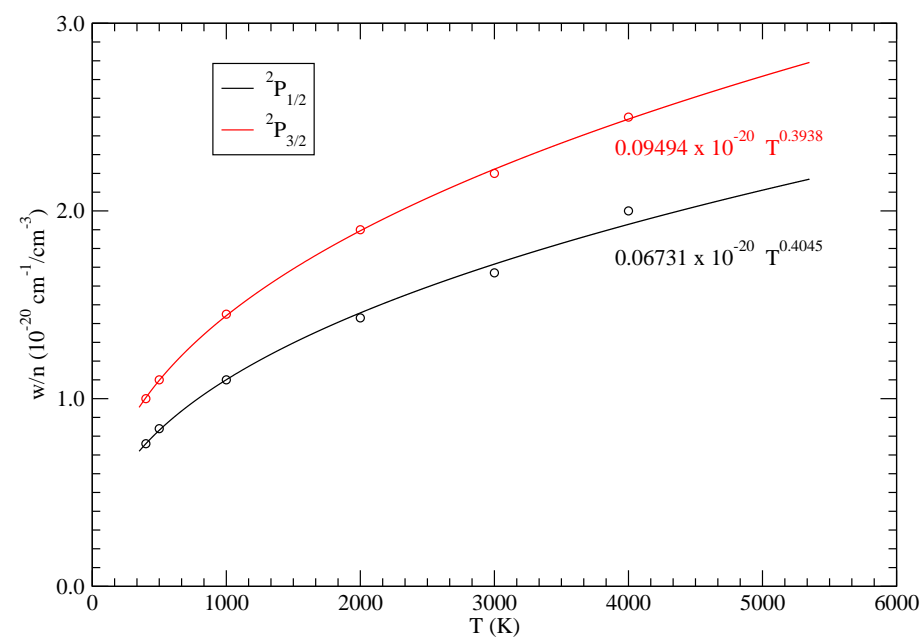

Figure 4: Widths (HWHM) of the Li resonance lines versus temperature follow a power law. The widths are linearly dependent on $\mathrm{He}$ density.

line width $(w)$ and are not accurately known from experiment, while the widths concur that the broadening rate is of the order of $1.0 \times 10^{-20} \mathrm{~cm}^{-2}$ at $600 \mathrm{~K}$. This value is in agreement with the new theoretical values, except that the experiments have not been able to independently measure the two lines of the doublet. The theory predicts that the transition to ${ }^{2} P_{3 / 2}$ will be broadened more.

\section{Experimental emission spectra}

Emission spectra of hot dense gases in a ballistic compressor were measured by Lalos and Hammond (1962) in a range of temperatures from 3600 to $6200 \mathrm{~K}$. They reported an unidentified possibly Li-He "violet" band at about $0.53 \mu \mathrm{m}$ which would agree with our calculation using the new molecular potential energies of Dell'Angelo et al. (2012). The fluorescence emission spectrum of the $\mathrm{Li}(2 s-2 p)$ resonance transition in the presence of helium at lower pressure and temperature $(670 \mathrm{~K})$ has been obtained by Scheps et al. (1975). They observed that the red wing consists of a broad plateau which extends to $0.9 \mu \mathrm{m}$ in agreement with our calculations of the emission profile for this temperature as shown in Fig. 5. These experimental results for the blue wing probe the potential energies at short range while the line core broadening and shift is a sensitive test of the long range interaction.

\section{Experimental absorption spectra at low temper- ature}

Similar to the interaction with a dense helium gas, the attachment of $\mathrm{Li}$ atoms to the surface of liquid helium droplets at temperatures around $380 \mathrm{mK}$ forms a similar environment and perturbation of alkali atomic transitions. Although in a different temperature regime, corresponding densities of surrounding helium atoms are comparable. 
Table 1: Half-width at half maximum (HWHM) $\left(10^{-20} \mathrm{~cm}^{-1} / \mathrm{cm}^{-3}\right)$ of Li resonance lines perturbed by He collisions

\begin{tabular}{llcccccc}
\hline Transition & Potential & $400 \mathrm{~K}$ & $500 \mathrm{~K}$ & $1000 \mathrm{~K}$ & $2000 \mathrm{~K}$ & $3000 \mathrm{~K}$ & $4000 \mathrm{~K}$ \\
\hline $2 s^{2} S_{1 / 2}-2 p{ }^{2} P_{1 / 2}$ & $s c$ & 0.76 & 0.84 & 1.1 & 1.43 & 1.67 & 2. \\
& $c c$ & & 1.01 & 1.34 & 1.76 & 2.07 & \\
$2 s^{2} S_{1 / 2}-2 p^{2} P_{3 / 2}$ & $s c$ & 1. & 1.1 & 1.45 & 1.9 & 2.2 & 2.5 \\
& $c c$ & & 1.01 & 1.34 & 1.77 & 2.09 & \\
\hline
\end{tabular}

$s c$ semi-classical calculations, this work, using ab initio potential energies of Dell'Angelo et al. (2012). $c c$ close-coupled calculations of Peach et al. (2006) using ab initio potential energies of Mullamphy et al. (2007).

Table 2: Experimental and theoretical line parameters, $w$ (HWHM) and $d,\left(10^{-20} \mathrm{~cm}^{-1} / \mathrm{cm}^{-3}\right)$ of the Li resonance lines perturbed by He collisions.

\begin{tabular}{llll}
\hline Reference & \multicolumn{1}{c}{ w } & d & T (K) \\
& & & \\
\hline Gallagher (1975) & $1.1 \pm 0.6$ & $-0.08 \pm 0.04$ & 400 \\
Smith and Collins (1976) & $0.92 \pm 0.05$ & $0.00 \pm 002$ & 520 \\
Lwin et al. (1977) & 1.01 & 0.03 & 600 \\
& & & \\
\hline
\end{tabular}




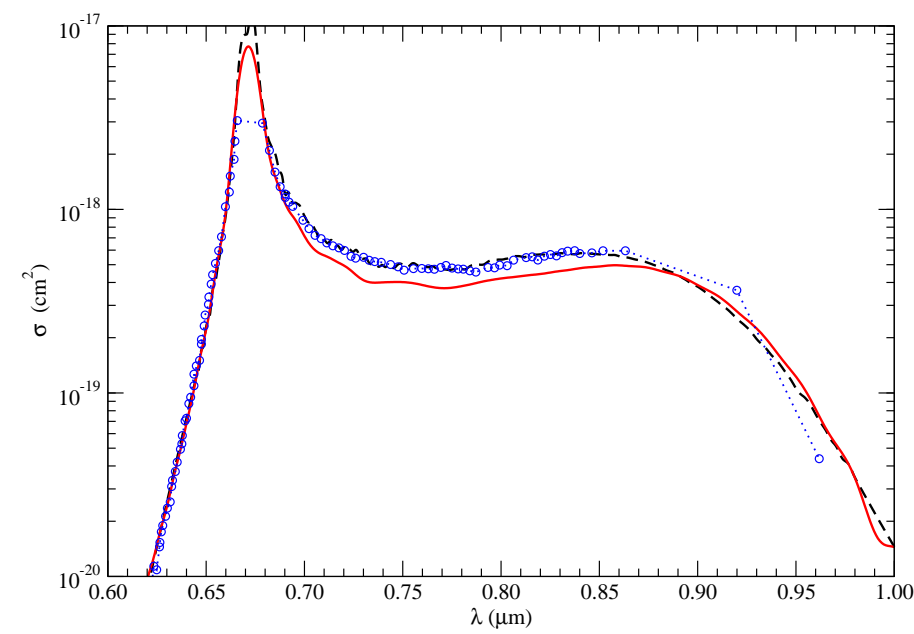

Figure 5: Emission spectra at $\mathrm{T}=670 \mathrm{~K}, n_{\mathrm{He}}=10^{19} \mathrm{~cm}^{-3}$. (red solid line Dell'Angelo et al. (2012), black dashed curve Pascale (1983), blue points Scheps et al. (1975)).

Experimentally, helium droplets are produced in a supersonic expansion and doped under high vacuum conditions with single Li atoms. Optical transitions have been characterized by means of laser-induced fluorescence excitation spectra (Stienkemeier et al., 1996; Bünermann et al., 2007). The interaction with the liquid helium surface leads to a characteristically broadened line profile (see Fig. 11). In particular, a pronounced blue wing is apparent.

\subsection{Li-He diatomic potential energies including spin-orbit coupling}

We now take into account spin-orbit coupling and consider the cores of the $D 1$ and $D 2$ components using the $a b$ initio Li-He potential energies of Dell'Angelo et al. (2012) shown in Figs. 6 and 7. We note that in Fig. 7 there is a shallow well with a depth of $1.5 \mathrm{~cm}^{-1}$ at $6 \AA$ in the ground state potential. It results in an increase of the modulated transition dipole moment $D(R)$ in the region of the minimum of $\Delta V(\mathrm{R})$ shown in Fig. 8.

The binding energy of the $\mathrm{X}^{2} \Sigma^{+}$state, that is the shallow ground state well, and the interaction energies of the excited states at long range, are all close to the limit of accuracy of these $a b$ initio calculations.

\subsection{Theoretical pressure dependence of the sum of the D1 and D2 components}

For a more direct comparison of the contributions of the two fine-structure components of the doublet it is convenient to use a cross section $\sigma$ associated with each component. The relationship between the computed cross section and the normalized absorption coefficient is:

$$
I(\Delta \omega)=\sigma(\Delta \omega) / \pi r_{0} f
$$

where $r_{0}$ is the classical radius of the electron, and $f$ is the oscillator strength of the transition.

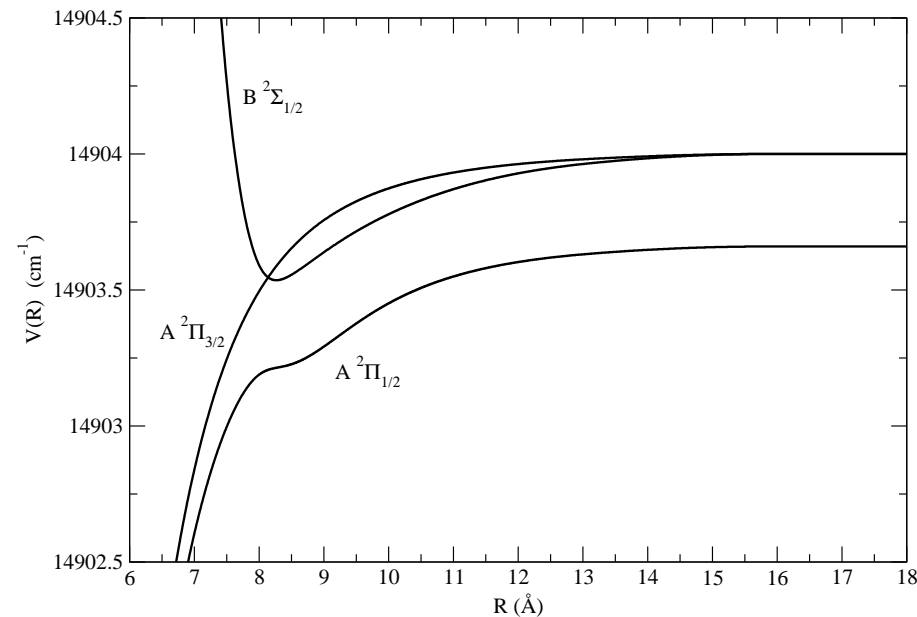

Figure 6: Potential energy curves for the $2 p$ state of LiHe molecule with spin-orbit coupling, ab initio potential energies are those of Dell'Angelo et al. (2012).

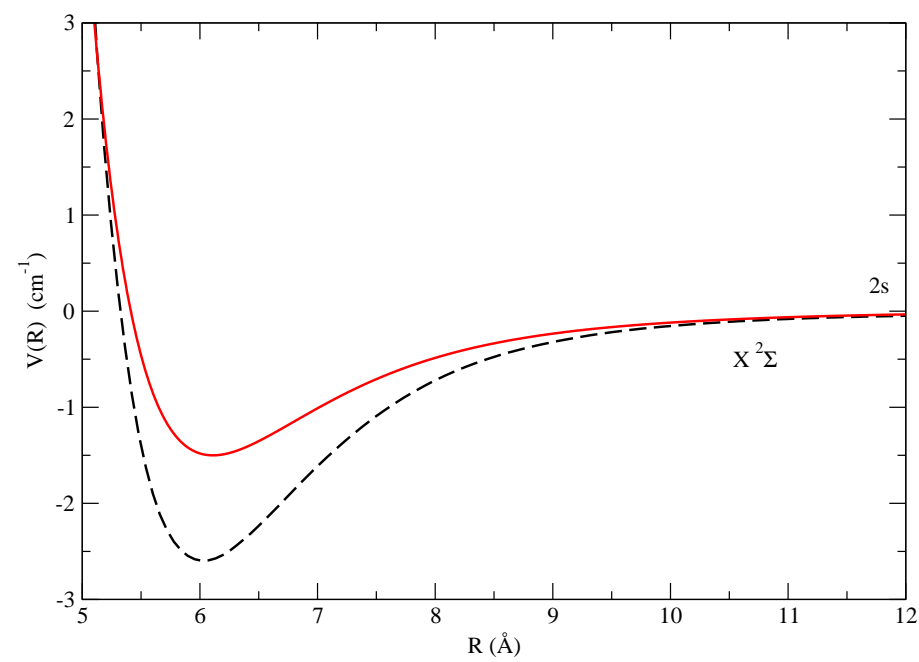

Figure 7: Potential energy curve for the $2 s^{2} \Sigma^{+}$state of Li-He (red solid line) Dell'Angelo et al. (2012). The Li-He potential energy (black dashed line) of Pascale (1983) is superimposed. 


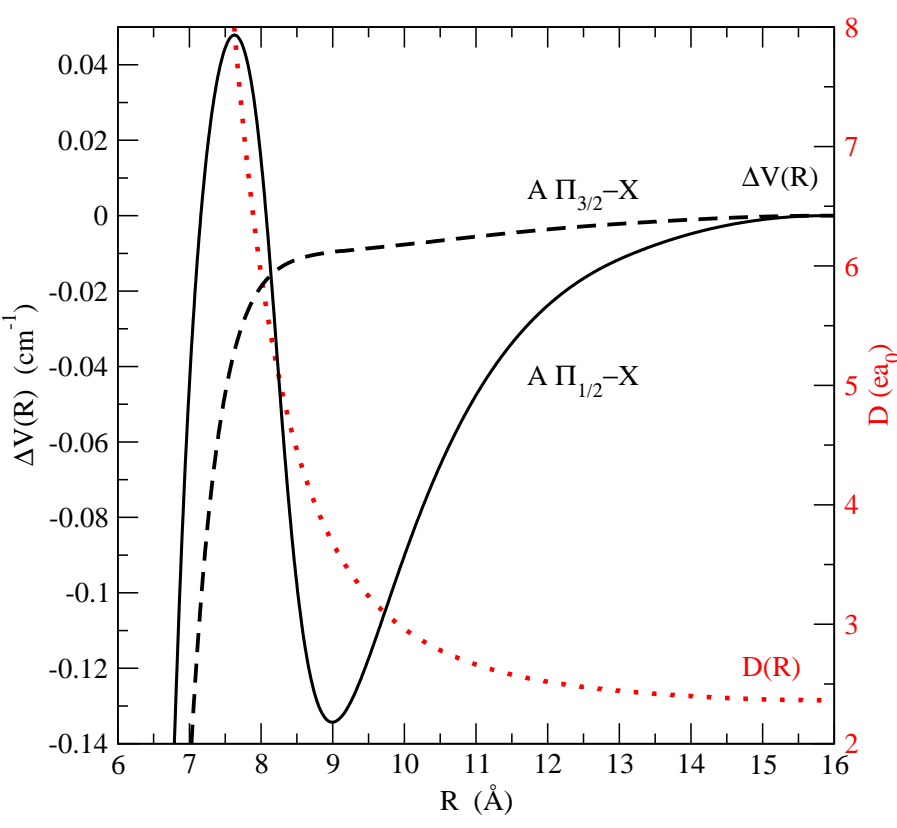

Figure 8: $\Delta V(\mathrm{R})$ (all black lines) for the $2 s X^{2} \Sigma^{+} \rightarrow 2 p A^{2} \Pi_{1 / 2}$ (full line) $2 s X^{2} \Sigma^{+} \rightarrow 2 p A^{2} \Pi_{3 / 2}$ (dashed line) transitions and $D(R)$, the $\mathrm{Li}-\mathrm{He}$ modulated dipole for $\mathrm{T}=0.38 \mathrm{~K}$ (dotted red line).

Absorption cross-sections for $2 s-2 p \mathrm{P}_{1 / 2}$ and $2 s-2 p \mathrm{P}_{3 / 2}$ transitions are shown in Figs. 9 and 10 . The two finestructure components are blended at these low temperatures and high densities. The amplitudes of the core the D1 and D2 lines decrease as He density increases as expected, but Fig. 10 also shows that the D1 line gains in strength relative to D2 in the core of the line whereas the strength in the blue wing of D2 remains unchanged. The D1 component is slightly shifted to the red while the D2 component is unshifted. The negative shift obtained in the unified profile shown in Fig. 11 is due to the critical long range part of the potential energy difference $\Delta V$ of the $2 s X^{2} \Sigma^{+} \rightarrow 2 p A^{2} \Pi_{1 / 2}$ transition. As can be seen in Fig. $8, \Delta V$ is very weakly attractive at long range $(R=9 \AA)$ with a well depth of $-0.134 \mathrm{~cm}^{-1}$. Since, as noted earlier, these values are close to the limit of accuracy of the $a b$ initio calculations, they are sensitive to the methodology or basis sets employed in the calculations.

It is well known that the shift of a spectral line profile is very sensitive to the long range part of the interaction potential, and for line shape calculations the accuracy of the potentials energies is crucial. An examination of Fig. 11 reveals that the width of the unified profile and the observed absorption spectrum are very similar, and serve as a confirming test of the intermediate range of the potential energies affecting the profiles under these conditions.

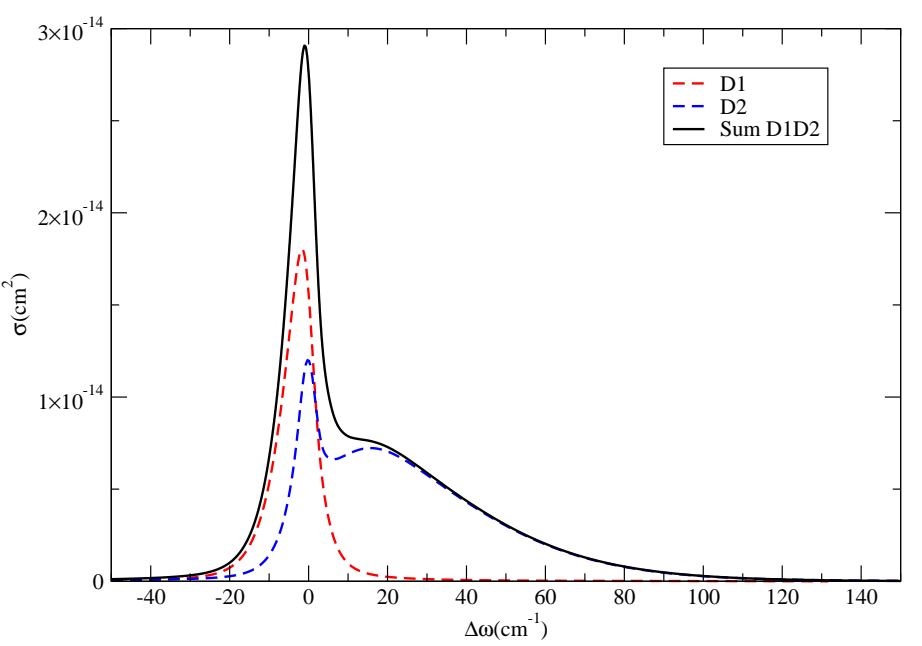

Figure 9: Comparison of the different contributions to the absorption spectrum at $\mathrm{T}=0.38 \mathrm{~K},\left(\mathrm{n}_{\mathrm{He}}=3 \times 10^{19} \mathrm{~cm}^{-3}\right) .\left(\Delta \omega=\omega-\omega_{0}\right.$, where $\omega_{0}$ is relative to the center of gravity of the two fine-structure components).

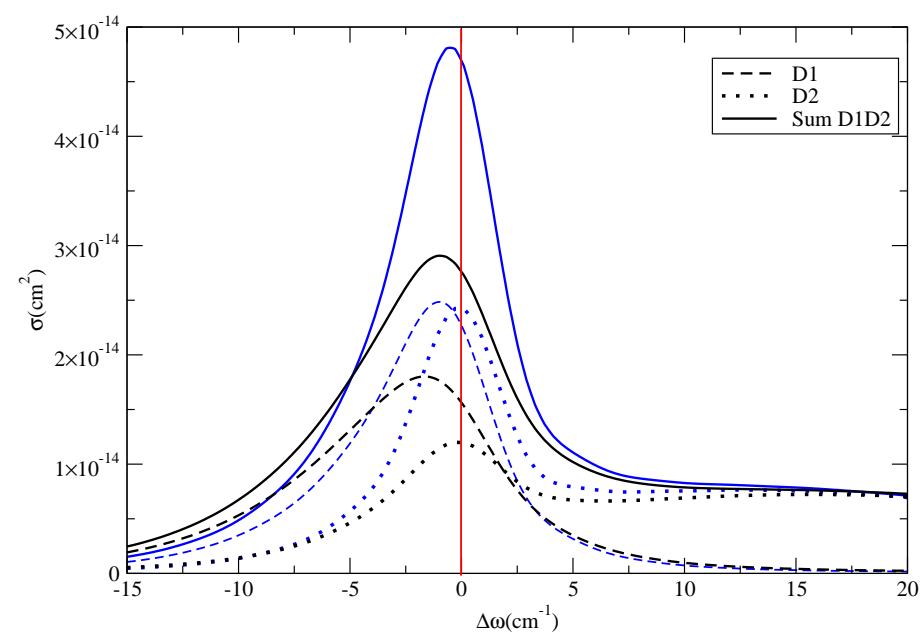

Figure 10: Variation with helium atom density of the absorption cross-sections for the D1 and D2 lines at $\mathrm{T}=0.38 \mathrm{~K}$. Black curves $\left(\mathrm{n}_{\mathrm{He}}=3 \times 10^{19} \mathrm{~cm}^{-3}\right)$. Blue curves $\left(\mathrm{n}_{\mathrm{He}}=2 \times 10^{19} \mathrm{~cm}^{-3}\right)$. $\left(\Delta \omega=\omega-\omega_{0}\right.$, where $\omega_{0}$ is relative to the center of gravity of the two fine-structure components). 


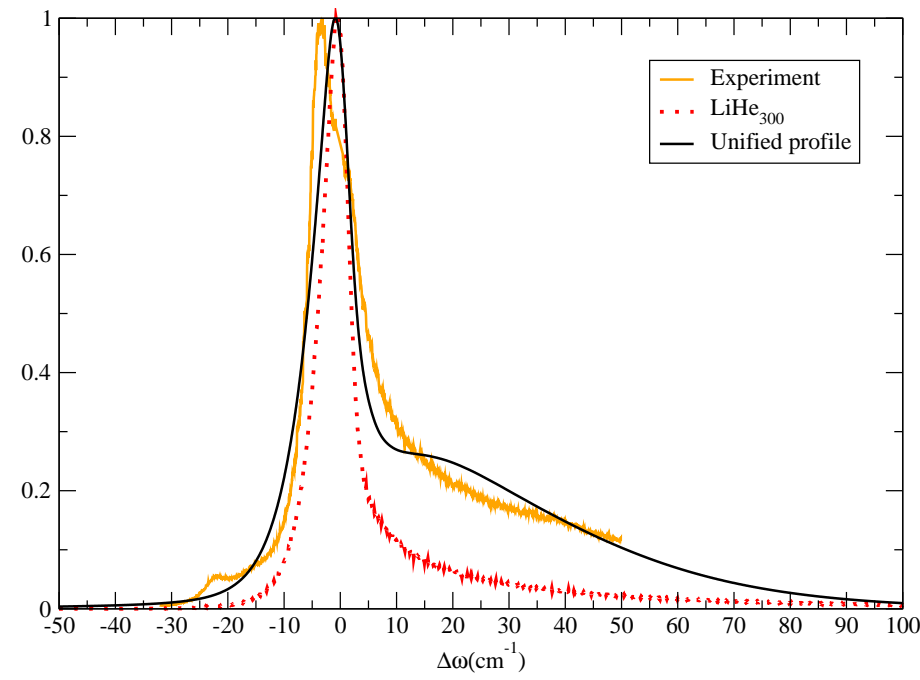

Figure 11: Unified profile at $\mathrm{T}=0.38 \mathrm{~K},\left(\mathrm{n}_{\mathrm{He}}=3 \times 10^{19} \mathrm{~cm}^{-3}\right)$ compared to PIMC calculations of Nakayama and Yamashita (2001) and experimental absorption spectrum of Li attached to large helium clusters $\mathrm{He}_{N}$. (Extracted from Stienkemeier et al. (1996)). $\left(\Delta \omega=\omega-\omega_{0}\right.$, where $\omega_{0}$ is relative to the center of gravity of the two fine-structure components).

\subsection{Comparison with experimental spectrum and path in- tegral Monte Carlo calculations}

Path integral Monte Carlo (PIMC) calculations have been performed at $\mathrm{T}=0.5 \mathrm{~K}$ by Nakayama and Yamashita (2001). The theoretical absorption spectra are shown in Fig. 11 compared with the experimental results. In our theoretical approach to the line shape, we use averages of independent Li-He pair collision processes, emphasizing the collisional process rather the collective quantum description of the interaction of $\mathrm{Li}$ with the He cluster in the PIMC theoretical approach.

Although the PIMC calculations of Nakayama and Yamashita (2001) do not exhibit the pronounced blue shoulder observed in the experiment the calculated profiles are similar in both theoretical models. They describe a radiating alkali atom surrounded by an equilibrium distribution of He atoms, and a spectrum given by multiple perturber phenomena in high density $\mathrm{He}$ at the very low temperature of liquid He (Allard et al., 2012, 2013). We notice that there is a close agreement of the collisional profiles with the experimental absortion spectrum in the wing shown in Fig. 11.

\section{Conclusion}

We offer an analytical expression for the impact broadened width of the resonance lines as a function of density and temperature. Combined with a unified line shape theory, new potential energies allow the computation of profiles that account for laboratory experiments over a wide range of $\mathrm{He}$ density and temperature, and that are expected to be useful under the conditions in substellar at- mospheres. We provide line broadening coefficients for the resonance line at temperatures up to $4000 \mathrm{~K}$ suitable for modeling the spectra of brown dwarf stars. Complete tables are available on request (from NFA).

We have shown that recent improvements to ab initio interatomic potential theory enable a calculation of the interactions of $\mathrm{Li}$ and $\mathrm{He}$ in the low lying atomic states over a range of atomic distances responsible for the broadening of the Li resonance lines. We would like to emphasize that the high accuracy at intermediate and long range internuclear distance of $a b$ initio potential energies obtained by Dell'Angelo et al. (2012) is crucial for the study of the line core of the D1 and D2 components observed at very low temperature described in Sect. 5.3. The success of these comparisons provides a stringent test of the validity of the potential energies, and confirms that the computed profiles will be valid for conditions of astrophysical interest.

\section{Citations}

Alioua, K., Bouledroua, M., 2006. Classical and quantal studies of the satellite features in the absorption spectra of lithium (2s-2p) perturbed by helium. Phys. Rev A 74, 032711-1 - 032711-7.

Allard, N.F., 1978. Alkali-rare-gas line profiles in a square-well potential approximation. I. Satellites. J. Phys. B: At. Mol. Opt. Phys. 11, 1383-1392.

Allard, N.F., Allard, F., Kielkopf, J.F., 2005. Theoretical profiles of light alkali resonance lines for brown dwarf atmosphere conditions. A\&A 440, 1195-1201.

Allard, N.F., Drira, I., Gerbaldi, M., Kielkopf, J.F., Spielfiedel, A., 1998. New study of the quasi-molecular Lyman alpha satellites due to $\mathrm{H}-\mathrm{H}$ and $\mathrm{H}-\mathrm{H}(+)$ collisions. A\&A 335, 1124-1129.

Allard, N.F., Kielkopf, J.F., Allard, F., 2007. Impact broadening of alkali lines in brown dwarfs. Euro. Phys. J. D 44, 507-514.

Allard, N.F., Koester, D., Feautrier, N., Spielfiedel, A., 1994. Freefree quasi-molecular absorption and satellites in Lyman-alpha due to collisions with $\mathrm{H}$ and $\mathrm{H}^{\wedge}{ }^{\wedge}{ }^{\wedge}$. A\&A Suppl. 108, 417-431.

Allard, N.F., Nakayama, A., Spiegelman, F., Kielkopf, J.F., Stienkemeier, F., 2012. Absorption spectra of NaHe from white dwarfs to helium clusters. Journal of Physics Conference Series 397, 012067. doi:10.1088/1742-6596/397/1/012067.

Allard, N.F., Nakayama, A., Spiegelman, F., Kielkopf, J.F., Stienkemeier, F., 2013. Absorption spectra of $\mathrm{Na}$ atoms in dense He. Euro. Phys. J. D 67, 52. doi:10.1140/epjd/e2013-30523-x.

Allard, N.F., Royer, A., Kielkopf, J.F., Feautrier, N., 1999. Effect of the variation of electric-dipole moments on the shape of pressurebroadened atomic spectral lines. Phys. Rev. A 60, 1021-1033.

Baranger, M., 1958a. Problem of Overlapping Lines in the Theory of Pressure Broadening. Phys. Rev. 111, 494-504.

Baranger, M., 1958b. Simplified Quantum-Mechanical Theory of Pressure Broadening. Phys. Rev. 111, 481-493.

Bünermann, O., Droppelmann, G., Hernando, A., Mayol, R., A.Stienkemeier, F., 2007. Unraveling the Absorption Spectra of Alkali Metal Atoms Attached to HeliumNanodroplets. J. Phys. Chem.A 111, 12684-12694.

Behmenburg, W., Makonnen, A., Kaiser, A., Rebentrost, F., Staemmler, V., Jungen, M., Peach, G., Devdariani, A., Tserkovnyi, S., Zagrebin, A., Czuchaj, E., 1996. Optical transitions in excited alkali + rare-gas collision molecules and related interatomic potentials: $\mathrm{Li}^{*}+\mathrm{He}$. Journal of Physics B Atomic Molecular Physics 29, 3891-3910. doi:10.1088/0953-4075/29/17/013.

Cohen, J.S., Schneider, B., 1974. Ground and excited states of Ne2 and $\mathrm{Ne}_{2}{ }^{+}$. I. Potential curves with and without spin-orbit coupling. J. Chem. Phys. 61, 3230-3239.

Cooper, D.L., 1982. Spin-orbit coupling and spin splitting in $\mathrm{HeNe}^{+}$. J. Chem. Phys. 76, 6443-6444. doi:10.1063/1.443003. 
Dell'Angelo, D., Guillon, G., Viel, A., 2012. Excited Li and $\mathrm{Na}$ in Hen: Influence of the dimer potential energy curves. J. Chem. Phys. 136, 114308. doi:10.1063/1.3693766.

Gallagher, A., 1975. Noble-gas broadening of the Li resonance line. Phys.Rev.A 12, 133-138. doi:10.1103/PhysRevA.12.133.

Lalos, G.T., Hammond, G.L., 1962. Emission Spectra of Hot Dense Gases. ApJ 135, 616-625. doi:10.1086/147294.

Lwin, N., McCartan, D.G., Lewis, E.L., 1977. The Determination of Collision Damping Constants. ApJ 213, 599-606. doi:10.1086/155191.

Mullamphy, D.F.T., Peach, G., Venturi, V., Whittingham, I.B., Gibson, S.J., 2007. Collisional broadening of alkali doublets by helium perturbers. J. Phys. B: At. Mol. Opt. Phys. 40, 1141-1152.

Nakayama, A., Yamashita, K., 2001. Path integral Monte Carlo study on the structure and absorption spectra of alkali atoms (Li, $\mathrm{Na}, \mathrm{K}$ ) attached to superfluid helium clusters. J. Chem. Phys. 114, 780-791.

Pascale, J., 1983. Use of l-dependent pseudopotentials in the study of alkali-metal-atom-He systems. The adiabatic molecular potentials. Phys. Rev. A 28, 632-644.

Peach, G., Gibson, S.J., Mullamphy, D.F.T., Venturi, V., Whittingham, I.B., 2006. Alkali Line Shapes for the Spectra of Brown Dwarfs, in: Oks, E., Pindzola, M.S. (Eds.), Spectral Line Shapes: XVIII, pp. 322-328. doi:10.1063/1.2402785.

Royer, A., 1974. Theory of pressure broadening in an adiabatic representation. Can. J. Phys. 52, 1816-1842.

Royer, A., 1978. Low-density approximations in the adiabatic theory of pressure broadening. Acta Phys. Pol. A 54, 805-822.

Royer, A., 1980. Shift, width, and asymmetry of pressure-broadened spectral lines at intermediate densities. Phys. Rev. A 22, 16251654 .

Scheps, R., Ottinger, C., York, G., Gallagher, A., 1975. Continuum spectra and potentials of Li-noble gas molecules. J. Chem. Phys. 63, 2581-2590.

Schneider, B., Cohen, J.S., 1974. Ground and excited states of Ne2 and $\mathrm{Ne}_{2}{ }^{+}$. II. Spectroscopic properties and radiative lifetimes. J. Chem. Phys. 61, 3240-3243. doi:10.1063/1.1682482.

Smith, G., Collins, B.S., 1976. Collision broadening and shift of the lithium resonance line by helium. Journal of Physics B Atomic Molecular Physics 9, L117-L120. doi:10.1088/0022-3700/9/5/011.

Stienkemeier, F., Higgins, J., Callegari, C., Kanorsky, S.I., Ernst, G., Scoles, W.E., 1996. Spectroscopy of alkali atoms (Li, Na, K) attached to large helium clusters. Zeitschrift fur Physik D Atoms Molecules Clusters 38, 253-263. doi:10.1007/s004600050090.

Zhu, C., Babb, J.F., Dalgarno, A., 2005. Theoretical study of pressure broadening of lithium resonance lines by helium atoms. Phys. Rev. A 71, 052710-1-052710-6. 